\title{
Síndrome coronariana aguda: risco em jovens
}

\author{
Acute coronary syndrome: risk in youth \\ Síndrome coronario agudo: riesgo en jóvenes
}

\author{
Leticia Pereira de Souza"; Renata Rigon ${ }^{I I}$; Maria Antonieta Moraes ${ }^{I I I}$; Silvia GoldmeierIV; Lúcia PellandaV
}

\begin{abstract}
RESUMO: O objetivo do estudo foi verificar o risco para síndrome coronariana aguda, em pacientes adultos jovens internados em um hospital de cardiologia do Rio Grande do Sul, entre setembro de 2010 e junho de 2012. Estudo transversal, com pacientes de 20 a 50 anos, ambos os sexos, com diagnóstico de síndrome coronariana aguda. Analisados fatores de risco cardiovasculares como: idade, história familiar positiva, tabagismo, índice de massa corpórea, atividade física, pressão arterial, glicemia, triglicerídeos, colesterol total e lipoproteína de alta densidade. Os resultados evidenciaram que dos 96 pacientes, $52(54,2 \%$ ) eram do sexo masculino, idade média de $43 \pm 7,4$ anos e $57(59,4 \%)$ com infarto agudo do miocárdio com supradesnivelamento do segmento ST. Os fatores de risco mais prevalentes foram: história familiar positiva 53(55,2\%) e tabagismo 49(51\%). A abordagem multidisciplinar deve ser instituída para modificação do estilo de vida, evitando ocorrência de novos eventos.
\end{abstract}

Palavras-Chave: Síndrome coronariana aguda; fatores de risco; adultos jovens; prevenção primária.

ABSTRACT: This cross-sectional study to determine risk for acute coronary syndrome in young adults examined patients of both sexes aged 20-50 years admitted to a cardiology hospital in Rio Grande do Sul State between September 2010 and June 2012 with diagnoses of acute coronary syndrome. The cardiovascular risk factors analyzed were: age, family history, smoking, body mass index, physical activity, blood pressure, glucose, triglycerides, total cholesterol and high density lipoproteins. The results comprised 96 patients, mean age $43 \pm 7.4$ years, $52(54.2 \%)$ of them male, and $57(59.4 \%)$ with acute myocardial infarction with ST-segment elevation. The most prevalent risk factors were positive family history 53(55.2\%) and smoking 49(51\%). A multidisciplinary approach should be introduced to modify lifestyle and prevent new events.

Keywords: Acute coronary syndrome; risk factors; young adults; primary prevention.

RESUMEN: El objetivo del estudio fue determinar el riesgo de síndrome coronario agudo, en pacientes adultos jóvenes internados en un hospital de cardiología de Rio Grande do Sul, entre septiembre de 2010 y junio de 2012. Estudio transversal realizado junto a pacientes de 20 a 50 años, de ambos sexos, con diagnóstico de síndrome coronario agudo. Se analizaron los factores de riesgo cardiovascular como: edad, historia familiar, tabaquismo, índice de masa corporal, actividad física, presión arterial, glucemia, triglicéridos, colesterol total y lipoproteína de alta densidad. Los resultados consistieron en 96 pacientes, $52(54,2 \%)$ hombres, promedio de edad $43 \pm 7,4$ años y $57(59,4 \%)$ con infarto agudo de miocardio y supradesnivelación del segmento ST. Los factores de riesgo más frecuentes fueron: historia familiar $53(55,2 \%)$ y tabaquismo $49(51 \%)$. El enfoque multidisciplinario debe ser instituido para la modificación de estilo de vida evitando la aparición de nuevos eventos.

Palabras Clave: Síndrome coronario agudo; factores de riesgo; adultos jóvenes; prevención primaria.

\section{INTRODUÇÃO}

Nos últimos anos, as doenças cardiovasculares (DCV) têm ocorrido, prematuramente, em adultos com idade inferior a 45 anos $^{1}$. A preocupação na área médica, social, psicológica e econômica tem sido relacionada à prevalência de doenças da sociedade atual, potencializada pelo estilo de vida pouco saudável. O diagnóstico entre os jovens não é tão bem caracterizado como em indivíduos mais velhos ${ }^{2}$. Os vasos comprometidos, muitas vezes, são únicos, o que pode estar relacionado a aspectos genéticos e história familiar de doença ${ }^{3}$.

Cerca de $5 \%$ a $10 \%$ do infarto agudo do miocárdio ocorre na idade adulta jovem ${ }^{4-6}$, compreendida entre $20 \mathrm{e}$

'Especialista em Cardiologia. Residência Multidisciplinar Integrada em Saúde: Cardiologia, Instituto de Cardiologia, Fundação Universitária de Cardiologia. Porto Alegre, Rio Grande do Sul, Brasil.E-mail: leticiapds@yahoo.com.br.

IIEspecialista em Cardiologia. Programa de Pós-Graduação Lato Sensu: Enfermagem em Cardiologia, Instituto de Cardiologia, Fundação Universitária de Cardiologia. Porto Alegre, Rio Grande do Sul, Brasil.E-mail: re_rigon@yahoo.com.br.

IIIDoutora em Ciências da Saúde: Cardiologia. Professora de Pós-Graduação Lato Sensu: Enfermagem em Cardiologia do Instituto de Cardiologia, Fundação Universitária de Cardiologia. Porto Alegre, Rio Grande do Sul, Brasil. E-mail: antonieta_moraes@uol.com.br.

${ }^{\text {IV }}$ Doutora em Ciências da Saúde: Cardiologia. Professora de Pós-Graduação Lato Sensu: Eñfermagem em Cardiologia do Instituto de Cardiologia, Fundação Universitária de Cardiologia. Porto Alegre, Rio Grande do Sul, Brasil. E-mail: pesquisa.sgold@gmail.com.

v'Cardiologista. Doutora em Ciências da Saúde: Cardiologia. Coordenadora do Programa de Pós-Graduação em Ciências da Saúde: Cardiologia, Fundação Universitária de Cardiologia. Porto Alegre, Rio Grande do Sul, Brasil. E-mail: lupellanda@gmail.com. 
40 anos, porém com melhores prognósticos ${ }^{5,6}$. Definindo esse perfil de risco é que se pode investir em prevenção primária, diminuindo gastos com reabilitação. Sendo assim, o objetivo deste estudo foi determinar os riscos em pacientes adultos jovens com síndrome coronariana aguda precoce, que se internam em um hospital-referência em cardiologia, no Estado do Rio Grande do Sul (RS).

\section{REFERENCIAL TEÓRICO}

Os fatores de risco (FR) que têm sido apontados como responsáveis pela maior prevalência de DCV nesta população são o tabagismo, hipercolesterolemia (modificáveis) e história familiar positiva (não modificável) ${ }^{5-8}$.

A exposição aos FR, normalmente, inicia-se na infância e consolida-se na juventude ${ }^{9-11}$. O depósito de colesterol nas artérias coronárias, desde a infância, prevê o aumento na extensão das lesões em idades mais avançadas ${ }^{12}$ e é por meio da cultura alimentar iniciada na infância que se poderá diminuir o avanço na progressão das lesões.

Estudos relatam que indivíduos que consomem maior quantidade de alimentos contendo gorduras saturadas possuem maior incidência de aterosclerose coronariana do que aqueles que não consomem. A longo prazo, as gorduras saturadas aumentam os níveis de colesterol e causam dano às coronárias ${ }^{13,14}$.

Outro aspecto de grande importância refere-se à prevalência da obesidade em nossa cultura. $\mathrm{O}$ alto consumo de alimentos industrializados e a prática de atividade física insuficiente são dois fatores que potencializam o surgimento da DCV no futuro. Recomenda-se a realização de, pelo menos, 30 minutos diários de intensidade leve ou moderada em cinco ou mais dias da semana ou, ainda, a prática de, pelos menos, 20 minutos diários de atividade física de intensidade vigorosa em três ou mais dias da semana. Reverter estilos de vida, considerados prejudiciais à qualidade de vida, torna-se um imperativo para as políticas públicas e práticas de saúde cuja orientação é focada na prevenção ${ }^{15,16}$.

A prevalência das dislipidemias em crianças e adolescentes vem crescendo no decorrer dos últimos anos, comprovada pela elevação dos níveis plasmáticos da lipoproteína de baixa densidade (LDL), fator decisivo no desenvolvimento da aterosclerose ${ }^{17}$.

Dados obtidos no RS, em 2008, evidenciaram 3.228 óbitos por doenças do aparelho circulatório. Destes, seis óbitos tinham entre 20 e 29 anos; 31 entre 30 e 39 anos e 150 entre 40 e 49 anos $^{18}$.

Estudos em diversos países demonstram preocupação com adultos que precocemente sofrem um evento isquêmico ${ }^{19-21}$.

Para que seja possível um planejamento com ações preventivas e eficazes, é necessário conhecer as particularidades dos FR da DCV na população-alvo.

\section{Metodologia}

Estudo transversal, desenvolvido de setembro de 2010 a junho de 2012, que incluiu pacientes adultos jovens com diagnóstico de síndrome coronariana aguda (SCA), hospitalizados em uma instituição de referência em cardiologia do Rio Grande do Sul.

Pacientes de ambos os sexos, com idade entre 20 e 50 anos com diagnóstico de SCA de qualquer etiologia foram incluídos no estudo. Pacientes que apresentaram barreiras de comunicação e portadores de doenças neurológicas degenerativas, instabilidade clínica ou que não aceitaram participar do estudo foram excluídos.

Um questionário com as variáveis: sexo, idade, história familiar positiva $(\mathrm{HF}+)$, hipertensão arterial sistêmica (HAS), hipercolesterolemia, hipertrigliceridemia, High Density Lipoproteins (HDL), tabagismo, sedentarismo, sobrepeso/obesidade e Diabete Mellitus (DM) foram utilizadas neste estudo.

Os casos considerados, neste estudo, como SCA, foram aqueles cujo diagnóstico médico foi de angina instável (AI), infarto agudo do miocárdio (IAM) com supradesnível de segmento ST (C/SST) e IAM sem SST. Todos os diagnósticos foram respaldados pelo eletrocardiograma (ECG) e pelo resultado de enzimas cardíacas alteradas (CK total para mulheres $>81 \mathrm{U} / \mathrm{L}$, e para homens > $129 \mathrm{U} / \mathrm{L}$; CK MB>10 $\mathrm{U} / \mathrm{L}$; Troponina T>100 pg/ml ${ }^{22}$. A HF+ foi considerada quando parentes de primeiro grau (pai, mãe ou irmão) tivessem desenvolvido alguma cardiopatia coronariana precocemente ${ }^{23}$. A hipertensão foi considerada quando a pressão arterial alcançou valores $\geq 140 / 90 \mathrm{mmHg}^{24}$. A Diabete Mellitus foi caracterizada com diagnóstico médico prévio, ou quando o paciente declarava uso de hipoglicemiantes orais e/ou insulina ou, ainda, quando duas glicemias de jejum consecutivas alcançavam valores $\geq 126 \mathrm{mg} / \mathrm{dl}$, ou uma delas media valores $\geq 200 \mathrm{mg} / \mathrm{dl}^{24}$. O colesterol total foi considerado quando valores plasmáticos alcançaram valores $\geq 240 \mathrm{mg} / \mathrm{dl}$, HDL alterado com valores $\leq 35$ $\mathrm{mg} / \mathrm{dl}^{25}$ e triglicerídeos elevados $(\geq 150 \mathrm{mg} / \mathrm{dl})^{25}$.

O tabagismo referiu-se a pacientes que fumavam qualquer quantidade de cigarro ao $\mathrm{dia}^{24}$. Sobrepeso significou índice de massa corpórea (IMC) $\geq 25 \mathrm{~kg} /$ $\mathrm{m}^{2}$; e obesidade $\mathrm{IMC} \geq 29,9 \mathrm{~kg} / \mathrm{m}^{2}{ }^{26}$. Sedentarismo foi avaliado mediante o questionário Internacional de Atividade Física (IPAQ-8) versão curta, validado no Brasil e proposto pela Organização Mundial de Saúde (OMS). Este contém questões sobre intensidade e duração da atividade física durante uma semana habitual, tanto em atividades ocupacionais quanto de locomoção, lazer ou prática esportiva, classificando os pacientes em: muito ativo, ativo, irregularmente ativo ou sedentário ${ }^{27}$. 
Para calcular a amostra, foi definida a prevalência de $50 \%$ da doença em pacientes com síndrome coronariana aguda, com margem de erro de $5 \%$ e intervalo de confiança de $90 \%$, resultando numa estimativa de 96 pacientes.

As análises foram realizadas utilizando o pacote estatístico Statistical Package for Social Sciences (SPSS) 19.0. As variáveis categóricas foram descritas em frequências absolutas e relativas (\%). As variáveis contínuas foram expressas em média e desvio-padrão ou mediana e percentis $25 \%$ e $75 \%$, conforme seguiram ou não distribuição normal.

Foi utilizado o teste quiquadrado para avaliar associação entre sexo e as variáveis qualitativas e, para as variáveis quantitativas, a comparação entre sexo foi utilizando-se o teste $\mathrm{T}$ ou o teste não paramétrico de Mann-Whitney. Foram aceitos como significativos valores de $\mathrm{P}<0,05$.

O projeto foi aprovado pela Comissão de Ética em Pesquisa do Instituto de Cardiologia/Fundação Universitária de Cardiologia, sob o número n ${ }^{\circ} 4516 / 10$. Os participantes que aceitaram participar do estudo assinaram um termo de consentimento livre e esclarecido (TCLE).

\section{Resultados E Discussão}

O sexo predominante foi o masculino $52(54,2 \%)$, com idade média de $43 \pm 7,4$ anos. Os FR mais prevalentes foram história familiar (+)53(55,2\%), tabagismo 49(51\%), sobrepeso/obesidade 70(71,8\%). Em relação aos exames bioquímicos, $17(17,7 \%)$ eram hipercolesterolêmicos, 44(45,8\%) com HDL $\leq 35 \mathrm{mg} / \mathrm{dl} \mathrm{e}$ $41(42,7 \%)$ estavam com triglicerídeos (TG) $\geq 150 \mathrm{mg} /$ dl. A glicemia aumentada esteve presente em $35(36,5 \%)$ sujeitos da amostra e a hipertensão em $24(25 \%)$.

A prevalência de IAM C/SST foi de $57(59,4 \%)$; $31(32,3 \%)$ apresentaram angina instável e $8(8,3 \%)$, IAM S/SST. O IAM C/ SST esteve presente em $57(59,6 \%)$ indivíduos do sexo masculino e $57(59,1 \%)$ do feminino, conforme registrado Tabela 1.

O nível da atividade física da amostra (IPAQ) está classificado em: muito ativos $14(14,6 \%)$; ativos $34(35,4 \%)$; os irregularmente ativos $35(36,5 \%)$ e os sedentários representando 13,5\%. Estes dados estão demonstrados na Figura 1.

TABELA 1: Prevalência da Síndrome Coronária Aguda segundo o gênero dos pacientes. Porto Alegre - RS, 2012. (N=96)

\begin{tabular}{lcc}
\hline Diagnóstico & $\begin{array}{c}\text { Masculino } \\
f(\%)\end{array}$ & $\begin{array}{c}\text { Feminino } \\
\mathrm{f}(\%)\end{array}$ \\
\hline Infarto agudo do miocárdio com supradesnivelamento do segmento st & $31(59,6)$ & $26(59,1)$ \\
Infarto agudo do miocárdio sem supradesnivelamento do segmento st & $6(11,5)$ & $2(4,5)$ \\
Angina instável & $15(28,8)$ & $16(36,4)$ \\
\hline
\end{tabular}

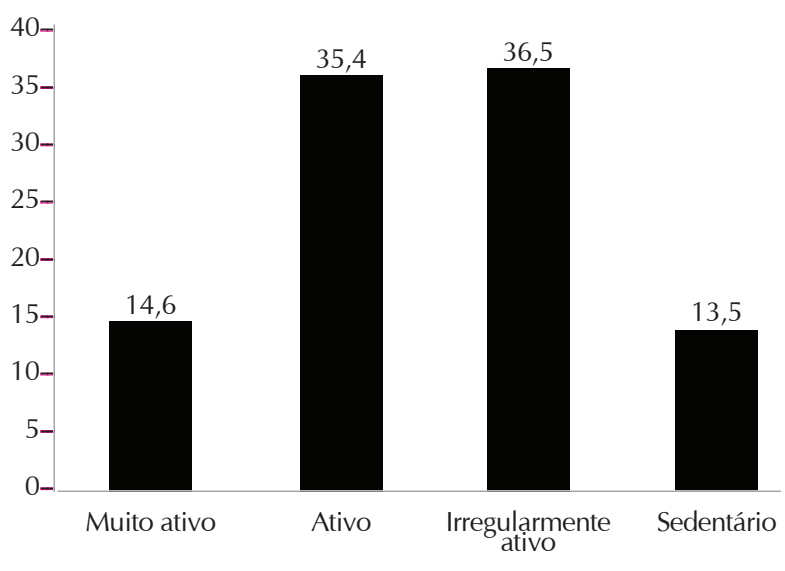

FIGURA 1: Distribuição percentual da atividade física classificada segundo IPAQ. Porto Alegre - RS, 2012. ( $N=96)$

\section{Discussão}

Objetivando demonstrar que os fatores de risco cardiovasculares e as DCV não estão presentes somente em fase da vida mais avançada, desenvolveu-se este estudo para identificar o perfil de risco em pacientes com SCA precoce, que se internam em um hospital universitário de referência em cardiologia no RS.

As características demográficas dos pacientes com SCA, neste estudo, demonstraram uma predominância do sexo masculino, com idade média de 43 anos. Esses achados vêm ao encontro de registros descritos na literatura, cuja prevalência de DAC ocorre com mais frequência em jovens do sexo masculino, tornando-se similares entre os gêneros por volta dos 80 anos $^{28}$.

Os fatores de risco encontrados na amostra com SCA evidenciaram a presença de história familiar positiva de DAC, seguida de tabagismo e sobrepeso. Em um estudo italiano conduzido com população da mesma faixa etária, os achados identificaram que a maioria dos indivíduos apresentavam história familiar positiva e tabagismo ${ }^{29}$.

A Organização Mundial da Saúde vem alertando que, embora o tabagismo tenha diminuído nos países desenvolvidos, ele está em ascensão de cerca de 3\% ao ano nos países em desenvolvimento. Pesquisa realizada pelo Sistema de Monitoramento de Fatores de Risco no Brasil mostrou que Porto Alegre (RS) e Rio Branco (Acre) são as capitais brasileiras com o maior percentual de fumantes: $21,2 \%$ do total da população ${ }^{30}$. 
Os resultados deste estudo evidenciaram elevada proporção de pacientes com sobrepeso e obesidade. Dados semelhantes foram registrados em diferentes cenários nacionais ${ }^{26}$ e internacionais ${ }^{31,32}$. Os consensos de prevenção secundária para DAC do American Heart Association e American College Cardiology consideram a obesidade como o maior fator de risco para doença cardiovascular ${ }^{33}$. Estudos referem uma maior reincidência de infarto e morte entre indivíduos com índice de massa corporal elevado ${ }^{34,35}$ e que a hipertensão é apontada como sendo um dos principais fatores de risco para $\mathrm{SCA}^{36}$. Na amostra da presente pesquisa, a maioria dos pacientes revelou níveis tensionais normais, resultado similar a um estudo com adultos cuja idade era menor do que 46 anos $^{19,21}$. Este fato é atribuído ao grande número de pacientes em uso de anti-hipertensivos há longo tempo. Além disso, esses resultados demonstram não ser um indicativo de normalidade nos valores da pressão arterial nessa faixa etária. A prevenção da hipertensão em fase precoce, por meio da prática de atividade física e alimentação com baixo teor de sódio, favorece os níveis tensionais normais.

Entre os indivíduos aqui investigados, os resultados demonstraram haver uma grande proporção de sedentários. Este achado mostrou um valor três vezes maior que um estudo paulista, no qual $6 \%$ da população estudada era sedentária e que $90 \%$ destes realizavam alguma atividade física recomendada como fator protetor às $\mathrm{DCV}^{26}$. Vale destacar que há diferença entre atividade física e exercício físico. A primeira é definida como qualquer movimento corporal, produzido pelos músculos esqueléticos, que resulta em gasto energético maior do que os níveis de repouso. Já o exercício físico significa uma atividade física planejada, estruturada e repetitiva, cujo objetivo final ou intermediário é o de aumentar ou manter a saúde/aptidão física ${ }^{37}$. Portanto, pode-se inferir que os pacientes deste estudo poderão ser considerados ativos do ponto de vista da atividade física, mas sedentários em relação à conceituação do exercício físico.

Valores glicêmicos alterados foram detectados em 34,3\% dos pacientes e o DM tipo II em 13,3\%. Tal diferença é explicada pelo critério do uso de insulina; alguns pacientes receberam insulina, conforme sua glicemia capilar, o que pode ter mascarado os exames laboratoriais. Da mesma forma, a média do perfil lipídico dos pacientes apresentou valores ideais, justificado pelo fato de a maioria dos pacientes estar em uso de medicamentos redutores de colesterol.

Embora a fase crítica da aterosclerose ocorrer principalmente na meia-idade, lesões ateroscleróticas já iniciam durante a infância. Além disso, alguns fatores de risco cardiovascular $(\mathrm{CV})$, como a hipercolesterolemia, hipertensão, tabagismo e obesidade, podem ter uma associação com o desenvolvimento de aterosclerose em adolescentes ou jovens adultos ${ }^{38}$. A detecção precoce de fatores de risco CV modificáveis nessa população pode ter um impacto em uma idade mais avançada. É necessário agir com medidas não farmacológicas e investir na prevenção, com uma alimentação saudável e a prática de exercício físico. A literatura ${ }^{29,31} \mathrm{faz}$, ainda, referência à importância do perfil lipídico, como um balizador da prevenção primária.

No entanto, os profissionais de saúde, entre os quais o enfermeiro, em sua prática de cuidar/educar/ pesquisar, devem se comprometer quanto ao provimento de informações que levem esta população à reflexão, à tomada de consciência das medidas a serem adotadas sobre seu estilo de vida.

\section{Conclusão}

Esta pesquisa fornece subsídios à busca de dados para um estudo prospectivo com um número maior de participantes. Os principais fatores de risco para síndrome coronariana aguda precoce entre os participantes foram: história familiar, tabagismo e sobrepeso. Destes, os fatores de risco modificáveis podem ser reduzidos, substancialmente, se houver investimento em promoção e prevenção das doenças e danos cardiovasculares responsáveis pelo aumento da morbimortalidade. Uma das formas de atuar na prevenção é incentivar as práticas de vida saudável na cultura familiar, fortalecendo as condutas individuais no futuro.

Entre as limitações deste estudo, destaca-se que outros fatores de risco, responsáveis pelo desenvolvimento da SCA, como presença do estresse, uso de álcool e hábitos alimentares não foram analisados, justificando a ausência da associação deles à SCA.

\section{REFERÊNCIAS}

1.Weinberger I, Rotenberg Z, Fuchs J, Sagy A, Friedmann J, Agmon J. Myocardial infarction in young adults under 30 years: risk factors and clinical course. Clin Cardiol. 1987; 10:9-15.

2.Maroszyǹska-Dmoch EM, Wożakowska-Kapłon B. Coronary artery disease in young adults: clinical and angiographic characterization. A single centre study. Kardiol Pol. 2015; 14:1-17.

3.Chouhan L, Hajar HA, Pompisiello JC. Comparison of thrombolytic therapy for acute myocardial infarction in patients aged $<35$ and $>55$ years. Am J Cardiol. 1993; 71:157-9.

4.Pasricha A, Batchelor W. When young hearts are broken: profiles of premature myocardial infarction. Am Heart J. 2002; 143:4-6.

5.Doughty M, Mehta R, Bruckman D, Das S, Karavite $\mathrm{D}$, Tsai T, et al. Acute myocardial infarction in the young - the University of Michigan experience. Am Heart J. 2002; 143:56-62.

6.Cantarelli MJC, Castello Jr HJ, Gonçalves R, Gioppato S, Navarro E, Guimarães JBF et al. Intervenção coronária percutânea em pacientes jovens. Rev Bras Cardiol Invasiva. 2014; 22:353-8. 
7.Avezum A, Makdisse M, Spencer F, Gore JM, Fox KA, Montalescot G, et al. Impact of age on management and outcome of acute coronary syndrome: observations from the Global Registry of Acute Coronary Events (GRACE). Am Heart J. 2005; 149:67-73.

8.Borba CS, Lemos IGS, Hayasida NMA. Epidemiologia e fatores de risco cardiovasculares em jovens adultos: revisão de literatura. Revista Saúde e Desenvolvimento Humano. 2015; 31:51-60.

9.Myers L, Coughlin SS, Webber LS, Srinivasan SR, Berenson GS. Prediction of adult cardiovascular multifactorial risk status from childhood risk factor levels. The Bogalusa Heart Study. Am J Epidemiol. 1995; 142:918-24.

10.Strong JP, Malcom GT, McMahan CA, Tracy RE, Newman WP, Herderick EE, et al. Prevalence and extent of atherosclerosis in adolescents and young adults: implications for prevention from the Pathobiological Determinants of Atherosclerosis in Youth Study. JAMA. 1999; 281:727-35.

11.Collins R, Peto R, MacMahon S, Hebert P, Fiebach NH, Eberlein KA, et al. Blood pressure, stroke, and coronary heart disease. Part 2, Short-term reductions in blood pressure: overview of randomised drug trials in their epidemiological context. Lancet. 1990; 335:827-38.

12.Theroux P, Fuster V. Acute coronary syndromes: unstable angina and non-Q-wave myocardial infarction. Circulation. 1998; 97:1195-206.

13.Keys A. The diet and 15-year death rate in the seven countries study. Am J Epidemiol. 1986; 124:903-15.

14.Krauss RM, Eckel RH, Howard B. AHA dietary guidelines: revision 2000: a statement for healthcare professionals from the Nutrition Committee of the American Heart Association. Circulation. 2000; 102:2284-99.

15.Santos I, Soares CS, Berardinelli LMM. Promovendo o autocuidado de clientes com obesidade e coronariopatia: aplicação do diagrama de pender. Rev enferm UERJ. 2013; 21:301-6.

16.Santos LR, Brito ECC, Neto JCGL, Alves LEP, Alves LRA, de Freitas RWJF. Análise do sedentarismo em estudantes universitários. Rev enferm UERJ. 2014; 22:416-21. 17.Leite AHP, Dantas TS, Rolim LADMM, Silva NQ, Morato CBA. Perfil lipídico em adultos jovens e fatores de risco associados a doenças cardiovasculares REBES.2015; $5(2): 15-20$.

18.Ministério da Saúde (Br). DataSUS [site de Internet]. Sistema de Informações sobre Mortalidade-SIM. [citado em 15 jan 2015]. Disponível em: http://portal.saude. gov.br/portal/-saude/profissional/visualizar_texto. cfm?idtxt $=32116 \&$ janela $=1$.

19.Tungsubutra W, Tresukosol D, Buddhari W, Boonsom W, Sanguanwang S, Srichaiveth B. Acute coronary syndrome in young adults: The Thai ACS Registry. J Med Assoc Thai. 2007; 90:81-90.

20.Aguiar C. Síndrome coronária aguda no jovem: essencialmente uma complicação aterotrombótica evitável. Rev Port Cardiol. 2010; 29:957-60.

21. Teixeira M, Sá I, Mendes JS, Martins L. Síndrome coronária aguda no jovem. Rev Port Cardiol. 2010; 29:947-55. 22.Keller T, Zeller T, Ojeda F, Tzikas S, Lillpopp L, Sinning $\mathrm{C}$, et al. Serial changes in highly sensitive troponin I assay and early diagnosis of myocardial infarction. JAMA. 2011; 306:2684-93.

23.Gus I, Fischmann A, Medina C. Prevalência dos Fatores de Risco da Doença Arterial Coronariana no Estado do Rio Grande do Sul. Arq Bras Cardiol. 2002; 78:478-83.

24.Sociedade Brasileira de Cardiologia, Sociedade Brasileira de Hipertensão, Sociedade Brasileira de Nefrologia. VI Diretriz Brasileira de Hipertensão. São Paulo: SBC; 2010.

25.V Diretriz brasileira de dislipidemias e prevenção da aterosclerose. Sociedade Brasileira de Cardiologia. Arq Bras Cardiol. 2013; 101(4 Supl.1):1-22.

26.Viebig R, Valero M, Araújo F, Yamada A, Mansur A. Perfil de saúde cardiovascular de uma população adulta da região metropolitana de São Paulo. Arq Bras Cardiol. 2006; 86:353-60.

27.Marshall A, Baumann A. The internacional physical activity questionnaire summary report of the reliability and validity studies. Geneva (Swi): IPAQ Excecutive Commite, World Heath Organization; 2001.

28.Schwartz JB, Zipes DP. CaBraunwald E, Bonow RO, Libby P, Zipes DP, editors. Braunwald's heart disease: a textbook of cardiovascular medicine. Philadelphia (USA): Elsevier Saunders; 2005.

29.Caimi G, Valenti A, Lo Presti R. Acute myocardial infarction in young adults: evaluation of the haemorheological pattern at the initial stage, after 3 and 12 months. Ann Ist Super Sanita. 2007; 43:139-43.

30.Ministério da Saúde (Br). Vigitel Brasil 2006: vigilância de fatores de risco e proteção para doenças crônicas por inquérito telefônico. Brasília (DF): Ministério da Saúde; 2007. 31.Rosengren A, Wallentin L, Simoons M, Gitt A, Behar S, Battler A, et al. Cardiovascular risk factors and clinical presentation in acute coronary syndromes. Heart. 2005; 91:1141-7.

32.DeVon H, Ryan C, Ochs A, Shapiro M. Symptoms across the continuum of acute coronary syndromes: differences between women and men. Am J Crit Care. 2008; 17:14-24. 33.Smith SCJr, Blair SN, Bonow RO, Brass LM, Cerqueira MD, Dracup K, et al. AHA/ACC Scientific Statement: AHA/ACC guidelines for preventing heart attack and death in patients with atherosclerotic cardiovascular disease: 2001 update. Circulation. 2001; 104:1577-9.

34.Rana JS, Mukamal KJ, Morgan JP, Muller JE, Mittleman MA. Obesity and the risk of death after acute myocardial infarction. Am Heart J. 2004; 147:841-6.

35.Kaplan RC, Heckbert SR, Furberg CD, Psaty BM. Predictors of subsequent coronary events, stroke, and death among survivors of first hospitalized myocardial infarction. J Clin Epidemiol. 2002; 55:654-64.

36.Conti R, Solimene M, Luz P, Benjó A, Neto P, Ramires J. Comparação entre homens e mulheres jovens com infarto agudo do miocárdio. Arq Bras Cardiol. 2002; 79:510-7. 37.Caspersen CJ, Powell KE, Christenson GM. Physical activity, exercise, and physical fitness: definitions and distinctions for health-related research. Public Health Rep. 1985; 100:126-31.

38.Jee-Aee Im, Ji-Won Lee, Jae-Yong Shim, Hye-Ree Lee, Duk-Chul Lee.Association between Brachial-Ankle Pulse Wave Velocity and Cardiovascular Risk Factors in Healthy Adolescents The Journal of Pediatrics. 2014 ; 150:247-51. 